\title{
Modeling the flow in nanostructured sensors within hybrid welding systems
}

\section{Sorin Vasile SAVU ${ }^{1, a}$, lonel Danut SAVU ${ }^{2, b}$ and Mihai GHIBA ${ }^{3, c}$}

1, 2, 3 Department of Engineering and Management of Technological Systems, Faculty of Mechanics, University of Craiova, Calugareni Street 1, 220037, Drobeta Turnu Severin, Mehedinti, Romania

asorin.savu@imst.ro, bdanut.savu@ymail.com, ’gomog44@yahoo.com

Keyword: nanostructured sensors, intelligent liquids, welding hybrid system

Abstract. This paper aims to present the recent results in modeling flows behavior in some specific nanostructured sensors. The sensor's concept is based on the advanced properties of the nanostructured materials. That is because the results in the field of the nanotechnologies showed that the sensors that are using advanced nanostructured materials are smaller, more precise, faster in response, and more efficient. The efficiency of such new type of sensor is claimed by some aspects which appear during the hybrid welding process.

\section{Introduction}

Modeling fluids behavior in a new type of sensor dedicated to the hybrid welding produces interesting features. The laser-arc hybrid welding is formed by two individual processes, laser welding and arc welding. The main advantage of this process is represented by the synergic effect obtained due to the putting together of the two heat sources. It means that the most important welding parameters are higher than the considered sum of the parameters of the two processes in individual consideration $[1,2]$

Intelligent flows with magnetic behavior - the so called "Ferro fluids" are ultra sensitive gel with magnetic properties, having three basic components [2]:

- basic liquid (in general any liquid);

- ultra smooth magnetic particles (in general $\mathrm{Fe}_{3} \mathrm{O}_{4}, \mathrm{Fe}$ or $\mathrm{Co}$ );

- strain - active stabilizing substance.

Because of the very small dimension of the magnetic particles $(3-20 \mathrm{~nm})$, of the large density of the gel particles $\left(10^{23} / \mathrm{cm}^{3}\right)$ and of the stabilizing layer in the basic liquid, the particles are integrating in the liquid structure, involving the properties of "intelligent fluid with magnetic behavior", even in the presence of a strong non uniform magnetic field, which generally destroy the stability of any usual gel. Liquid (silicone oil, mineral oil, etc) based fluids mixed up with surfactants and magnetic particles with dimensions ranging between $0.1 \mu \mathrm{m}$ and $100 \mu \mathrm{m}$ are know under the name of fluids or/and magneto rheological suspensions (MRS). A remarkable property of MRS is that its fluidity can be modified from the value of the intensity of an external magnetic field. This property finds utilizations in various applications. MRS mixed up with graphite microparticles, modifies its conductivity function of the intensity and direction of the magnetic field intensity vector. Based on this, property sensors with destinations specified were achieved in MRS. Thus the suspension structure will modify step by step when an alternant magnetic field is applied. In figure 1 it is observed that change for $1 \mathrm{~s}, 3 \mathrm{~min}, 15 \mathrm{~min}$ and 1 hour period since the magnetic field is applied.

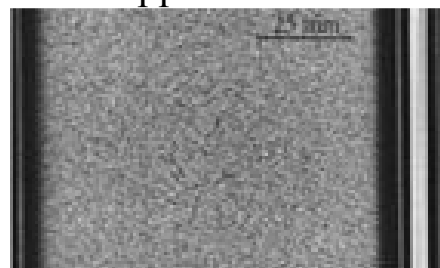

$\mathrm{t}=1 \mathrm{~s}$

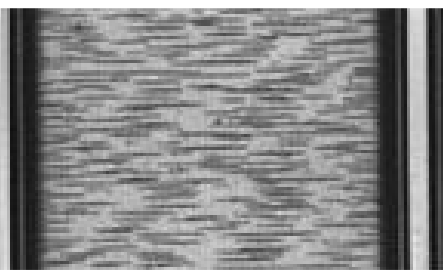

$\mathrm{t}=180 \mathrm{~s}$

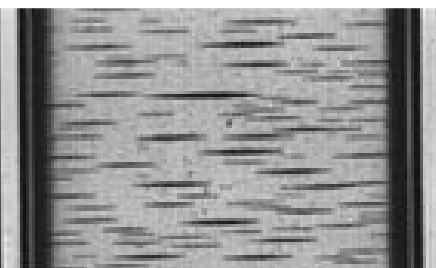

$\mathrm{t}=900 \mathrm{~s}$

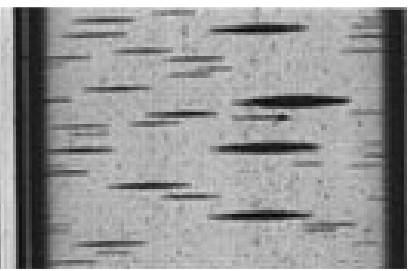

$\mathrm{t}=3600 \mathrm{~s}$

Fig. 1 MRS fluids in alternant magnetic field 
It is shown that the thickness of the oil layer between the magnetic dipoles oriented according to the magnetic field lines, defines the value of the MRS electric conductivity. Production of MRS is achieved by means of an experimental installation and a method, both described in references. A mixture consisting of:

- $\mathrm{Fe}_{2}(\mathrm{CO})_{9}$ powder with granulation of (4.5-5.2) $\mu \mathrm{m}$, its quantity being $0.082 \mathrm{~kg} \pm 5 \%$;

- Aneron/Merck type mineral oil, its quantity being $0.025 \mathrm{~kg} \pm 5 \%$

- Stearic acid, its quantity being $0.002 \mathrm{~kg} \pm 2 \%$

The mixture so formed is homogenized and treated thermal for $1,800 \mathrm{~s}$ at the temperature of $510^{0}$ $\mathrm{K} \pm 10 \%$. Using this fluid, a gravitational nanostructured sensor has been designed. Figure 2 presents the constructive solution of the sensor. Inside a circular tube which has a torus form, a nanofluid is introduced for $1 / 3$ length of the tube. On the rest of the tube 2 coils are fixed. The liquid fills up about a tierce of torus circumference, the other two tierces being filled by the bobbins. The position of $\beta=45^{\circ}$ is considered the reference position, when the intelligent liquid belongs outside the bobbins fixed onto the torus. The same domain, $\pm 30^{\circ}$, was taken into account for this sensor too, being adequate for all technical variations.

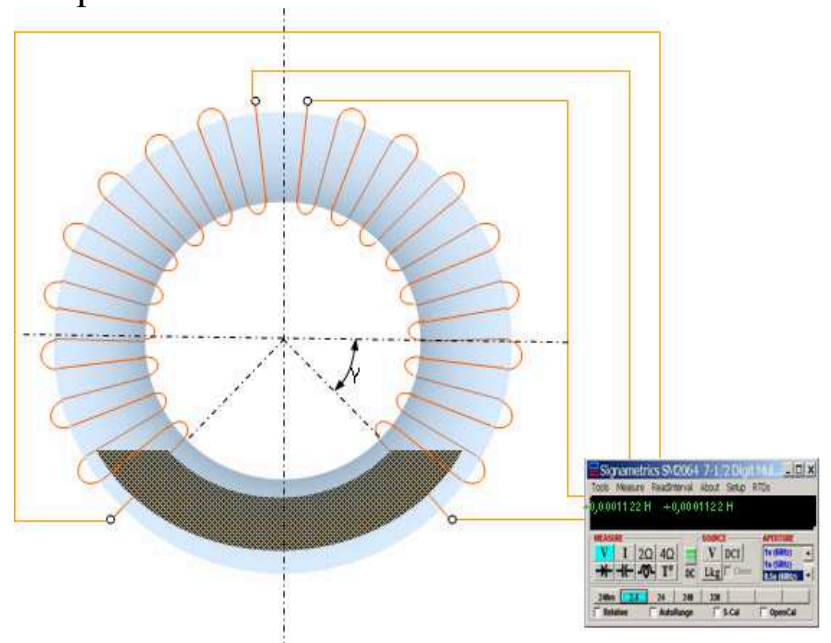

Fig. 2 The compact design of a gravitational nanostructured sensor

An important phenomenon which is observed during the sensor activity is the liquid circulation into the torus. The circular moving given by the torus during the sensor activity implies that the magnetic liquid is slowly introduced inside the coils, the magnetic inductance being linear [3]. By highly precision measurement (micro-Henry range) the functioning curve is built (Figure 3), representing the variation of the measured inductance versus the angle of rotation.

$\mathrm{L}[\mathrm{mH}]$

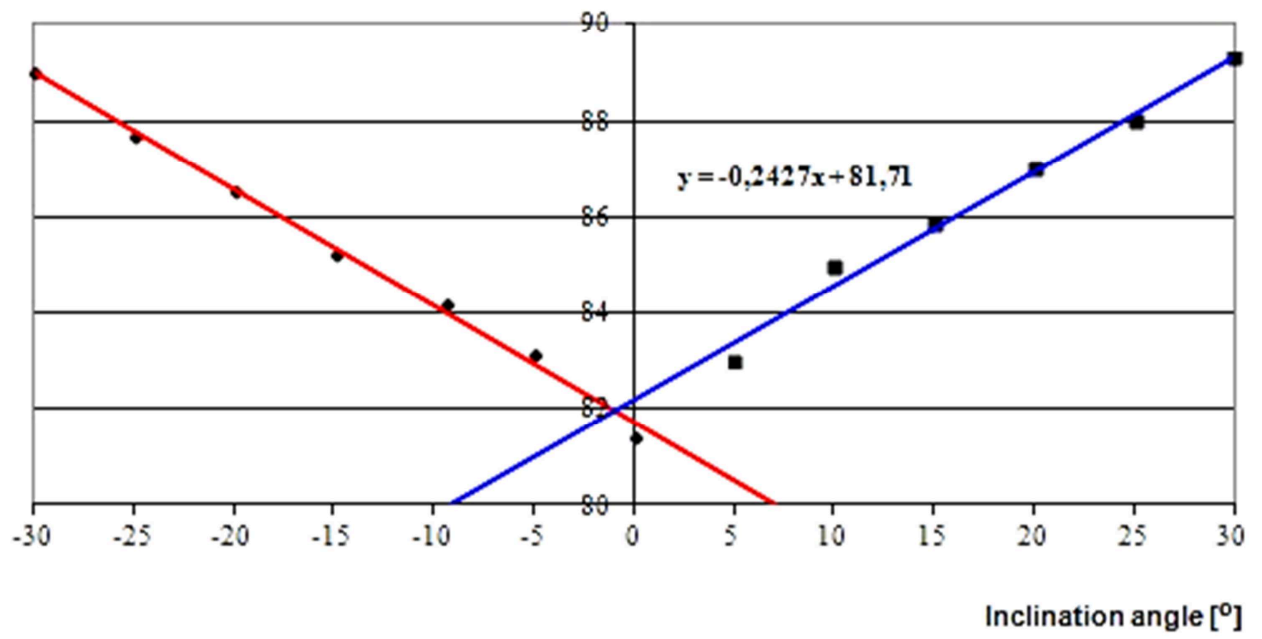

Fig. 3 Functioning curve of the nanostructured sensor 
Each of the two coils has two terminals connected to a data acquisition system. The absorbed signal is the momentum inductance of the coil. Without magnetic core, the coil has its own inductance, determined by its building features. Introducing slowly the liquid into the coil, the inductance grows from initial value to a specific value determined by the quantity of magnetic liquid introduced as core. This value is discretely taken up by the data acquisition system and introduced in a numeric calculus system ( $\mathrm{PC}+$ software) which extracts the initial value of the coil inductance. Thus, an absolute inductance value is obtained for a certain quantity of magnetic liquid introduced in the core.

\section{Modeling the behavior of the magnetic liquid within the torus}

An interesting analysis of the fluid flow within the torus can be realized by an energetic approach. An adequate flow (in which the liquid clings slowly at the torus walls) is a flow with minimum energy breakage. The optimization is based on the analysis of the energy functional variation with respect of the center angle variation. In this order the integral form of the energy is considered, namely:

$$
E=\frac{1}{2} \int_{\Omega}|v|^{2} d x
$$

The approach is characterized by:

- Is the three-dimensional integration case, $\Omega$ being the integration domain, in our case the torus channel;

- Since $\mathbf{v}=\mathbf{v}(\mathbf{x}, t)$, the modeling method consists in studying the energy variation in the flow process, when there are a strip of values both for the two torus radius and for the center angles.

- For the simulations the soft MAPLE11 was used, as follows: MultiInt procedure for evaluating the integral, and InteractivePlot for plotting the variation of the energy for few numerical situations.

- Since there is a numeric value for the starting velocity, a polynomial expression is taken into account, implying also the time parameter

\section{Results and discussion}

The simulations referred to the gravitational flow specific to the gravitational sensor. There were taken into account two values sets for the sensor. The integration domain will be given by the equation 2 and the center angle has been considered to be $30^{\circ}$. The simulation of the conditions is presented below:

- The outer torus radius $\mathrm{R}=13 \mathrm{~mm}$, the inner radius $\mathrm{r}=10 \mathrm{~mm}$ without flow velocity coefficient.

$$
R_{1}=\frac{R-r}{2}=11,5 \mathrm{~mm}
$$




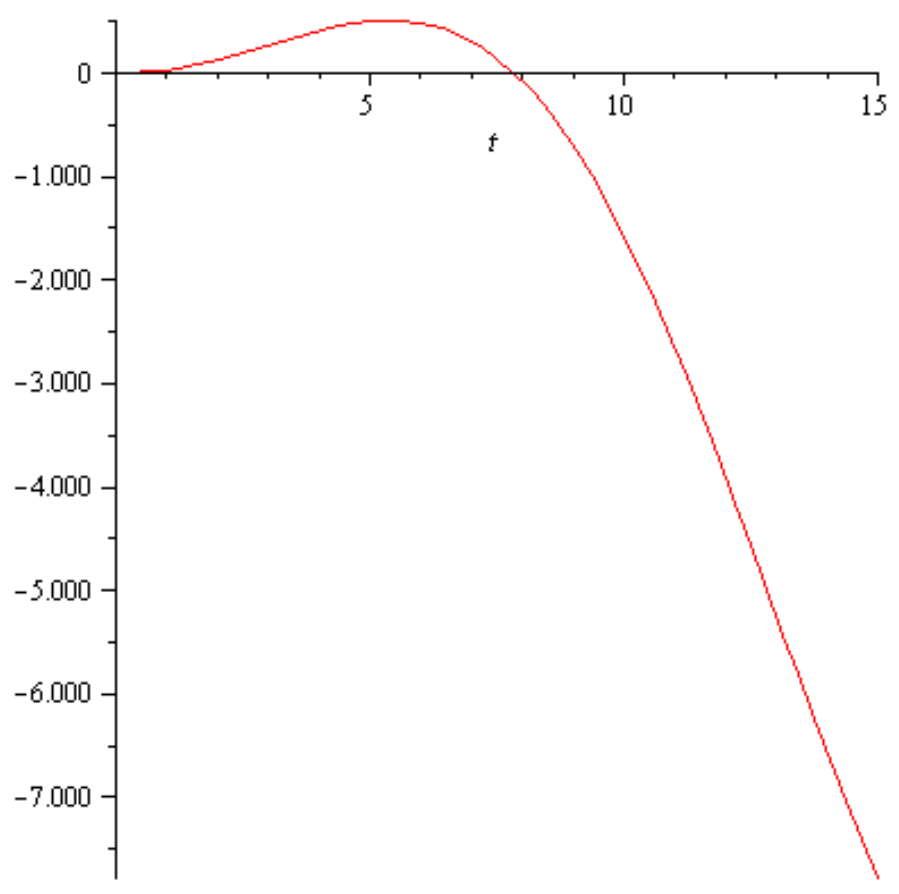

Fig. 4 First case of simulation

- The outer torus radius $\mathrm{R}=13 \mathrm{~mm}$, the inner radius $\mathrm{r}=10 \mathrm{~mm}$ with flow velocity coefficient which can be approximated using three equations as follows:

$v=23+t^{2}$

$v=23-t^{2}$

$v=23 \times t^{2}$

Where the coefficient " 23 " is given by the equation 6 presented below:

$\omega \times R_{1}=2^{0} \times 11.5$

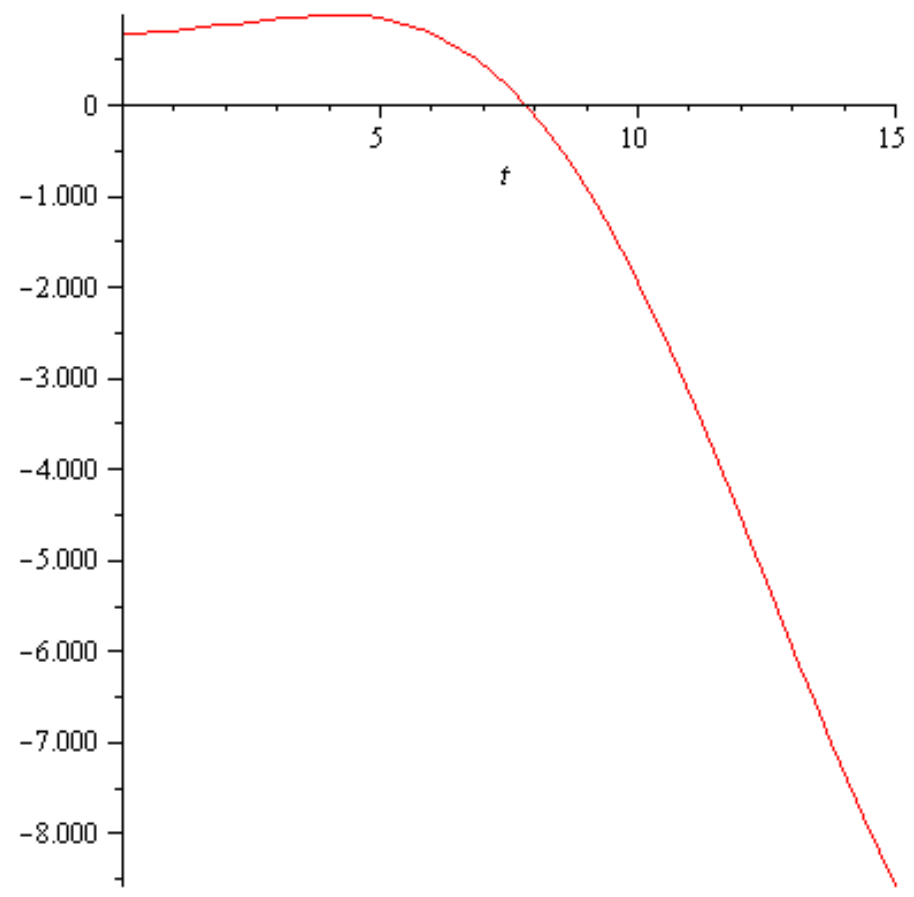

Fig. 5 The second case of the simulation 


\section{Conclusions}

Few remarks are available related to the previous simulations:

- Related to the first case - two features have to be outlined:

- The simulation offers a concrete answer, but from practical standpoint it cannot be deduced the evolution of a flow with a fast depletion. This depletion is proved by the decreasing of the energy;

- The model does not take into account the geometric elements of the torus. Therefore a future analysis should take into account also this feature;

- Concerning the second simulation case, the geometric elements of the torus were taken into account. Although the addition sign "+" is not quite proper from technical standpoint, a real image is obtained for the first 10 seconds of magnetic liquid flow.

- There were also tested another simulation sub-case of this case, it was observed an increasing and then a decreasing of the energy, and also some simulation errors beginning with the 17 second of the simulation (figure 6)

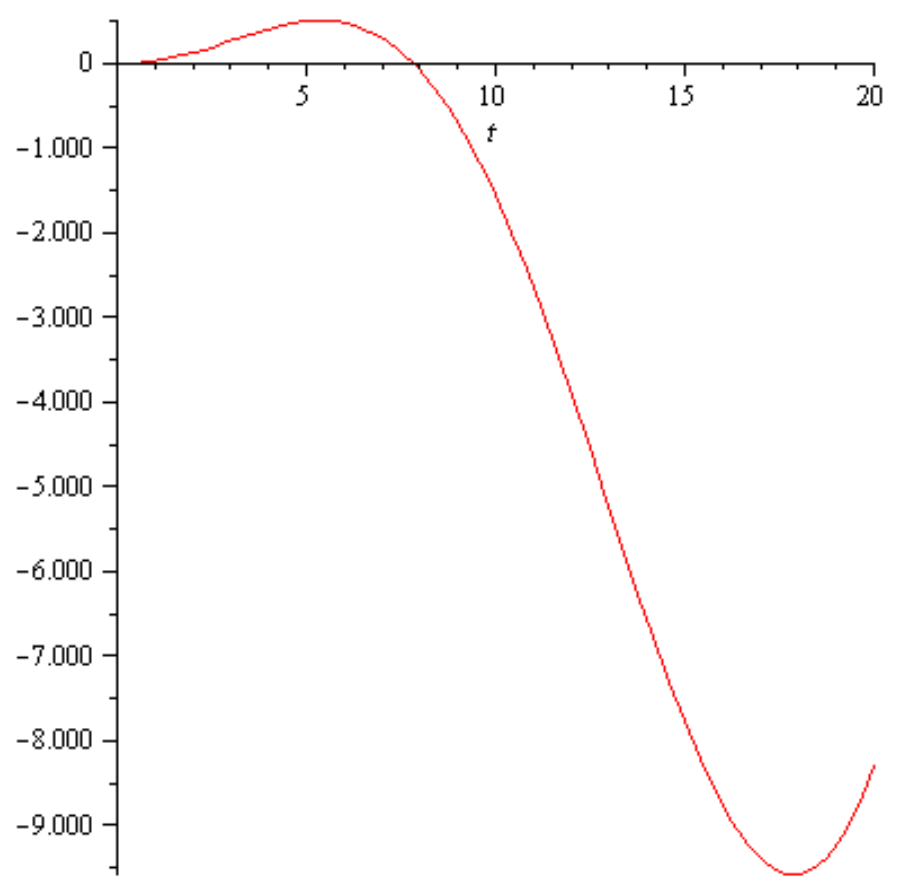

Fig. 6 The second situation of the first simulation $(\mathrm{t}=0-20 \mathrm{~s})$

The above models, together with the other simulation cases, offer a quite real approach of the experiments, therefore the models could be considered good. Actually, there were noticed cases when the model functioned only for short periods, therefore a different computational standpoint should improve the simulation analysis. This is a next aim.

\section{Acknowledgement}

This work was partially supported by the grant number $47 \mathrm{c} / 2014$, awarded in the internal grant competition of the University of Craiova 


\section{References}

[1] Sîrbu N. A., Şerban V.A., Stan D., Oancă O. - Ultrasonic Device for Processing Polymer Composite Materials, ModTech International Conference, Modern Technologies, Quality and Innovation - New face of TMCR, 25-27 May 2011, Vadul lui Voda, Chisinau, Republic of Moldova

[2] A. Ionescu, I.D. Savu, S.V. Savu, D. Coman - Computational features of flow modeling in nanostructured sensor, BICS 2008: Proceedings of the 1st International Conference on Bio-Inspired Computational Methods Used for Difficult Problems Solving: Development of Intelligent and Complex Systems. AIP Conference Proceedings, Volume 1117, pp. 125-132 (2009), DOI: http://dx.doi.org/10.1063/1.3130614

[3] Bîrdeanu A.V., Ciucă C., and Puicea A. - Pulsed LASER-(micro)TIG hybrid welding: Process characteristics," J. Mater. Process. Technol., 212(4), pp. 890-902.

[4] V. Verbițchi, A.V. Bîrdeanu, N.A. Sîrbu - Voltage-frequency converter for laser welding control, International Journal of Electronics,pp1 8, DOI:10.1080/00207217.2012.743065, dec.2012, Publisher: Taylor \& Francis, Informa Ltd Registered in England and Wales Registered Number: 1072954 Registered UK

[5] Ciupitu, I., et al. - The improving of the process of the iron, the cast iron and the copper powder mixing, Materials Science Forum Vol. 672 (2011), pag. 76-79, edited by Trans. Tech. Publications Ltd, Switzerland, ISSN: 1662-9752, DOI: 10.4028/www.scientific.net/MSF.672.76

[6] Bîrdeanu V., Dehelean D., Harjan I., and Ciucă C., 2010, "Technological system for studying the pulsed LASER-microTIG hybrid welding process dynamics," Bull. Polytech. Inst. Iași, LVI(2a), pp. 49-58. 